\title{
Geography of racial coresidence
}

\author{
Anna Dmowska ${ }^{\mathrm{a}, \mathrm{b}}$, Tomasz F. Stepinski ${ }^{\mathrm{a}}$ \\ ${ }^{a}$ Space Informatics Lab, Department of Geography and GIS, University of Cincinnati, Cincinnati, USA, OH 45221-0131, USA \\ ${ }^{b}$ Institute of Geoecology and Geoinformation, Adam Mickiewicz University, Poznan, Poland
}

\begin{abstract}
Whereas most work on residential race relations in US cities is based on the concept of segregation, our approach studies this issue from a who-lives-with-whom perspective. To this end, we study coresidence profiles - percentages of a given racial subpopulation living in different population zones. Population zones are data-driven divisions of a city based on characteristic racial compositions. We used 1990 and 2010 decennial census block-level data for 61 largest US metropolitan areas to calculate coresidence profiles for four major racial subpopulations in each city at both years. Profiles for each race/year combination were clustered into three archetypes. Cities, where given race profiles belong to the same archetype, have similar coresidence patterns with respect to this race. We present the geographic distributions of co-habitation profiles and show how they changed during the 1990-2010 period. Our results revealed that coresidence profiles depend not only on racial preferences but also on the availability of racial groups; cities in the different geographical regions have different coresidence profiles because they have different shares of White, Black, Hispanic, and Asian subpopulations. Temporal changes in coresidence profiles are linked to the increased share of Hispanic and Asian populations.
\end{abstract}

Keywords:

coresidence, racial distribution, residential segregation, census, spatial inhomogeneity

\section{Introduction}

Major cities in the United States (US) are inhabited by people of different races and ethnicities. Over the last century, the racial composition of those cities has been shaped by two waves of migration - a Great Migration (1916-1970) and an international migration from Latin America and Asia (since the 1970s). It is estimated that during the Great Migration 6.6 million African Americans moved from rural southern US to northern and Midwest industrial cities (Frey, 2004). The Great Migration significantly influenced the racial composition of major American cities changing them from monoracial to bi-racial. The second time the racial composition of major cities has been significantly affected was by the influx of immigrants from Latin America and Asia which started in 1970 and is ongoing. According to the 2010 Census, the Asian population is the fastestgrowing racial subpopulation in the US. Asians are a highly urbanized population, concentrated mostly in the West and South region (Hoeffel et al., 2012). Hispanics

*Corresponding author. Email address: stepintz@uc.edu are the second-largest ethnic group in the United States concentrated mostly in the West and South regions, with over half of the Hispanic population in the US residing in California, Texas, and Florida (Humes et al., 2011). Hispanics and Asian populations have contributed to an important demographic change in the United States since the 1970s, changing the racial composition of US cities from bi-racial to multiracial (Iceland, 2004).

Because these waves of migration affected multiple aspects of US society (demographic, political, and economic), there is a significant body of literature pertaining to issues of racial diversity and segregation in US cities, as well as to their changes over time. The vast majority of studies concentrate on the topic of urban residential segregation. In those studies city's segregation is encapsulated by a single number - a segregation index (Massey and Denton, 1987; Iceland et al., 2002; Iceland, 2004; Farrell, 2008; Fischer et al., 2004; Logan et al., 2004; Fischer, 2003; Parisi et al., 2011; Fowler et al., 2016; Ellis et al., 2018; Bellman et al., 2018). A segregation index (there are multiple versions of segregation index (Hong et al., 2014; Yao et al., 2019)) is a convenient tool to compare the overall magnitude of 
segregation between different cities or between a single city at different times. However, because it integrates information into a single number, such an index tells us very little about particulars, such as which races are highly segregated and which are more integrated. It also does not tell us about observed preferences for racial integration (Farley et al., 1978; Clark, 1992; Charles, 2000; Havekes et al., 2016).

The minority of past studies took a more in-depth approach in which individual neighborhoods within a city (assumed to coincide with census tracts) are classified into different types of their racial character based on the local racial mix. Several different classification schemes were proposed, Fasenfest et al. (2004), Farrell and Lee (2011), and Logan and Zhang (2010) based their classifications on racial composition, Holloway et al. (2012) and Wright et al. (2014) used levels of racial diversity and dominant race, while Bader and Warkentien (2016) and Clark et al. (2015) used more complex statistical techniques.

Regardless of the classification method, a racial description of the city is provided by a histogram (shares of tracts of different types among all tracts in a city). Inter-city comparison comes down to a comparison of two histograms and temporal change is assessed by calculating a transition matrix (counting a number of tracts that changed from a given racial type at an earlier year to another type in a later year). Although the neighborhood-based classification approach provides more in-depth information than the segregation index, its reliance on counting tracts fails to provide information about the area of the city or the number of people affected by the change. It also does not provide insight into racial residential preferences.

The goal of this paper is to characterize residential relations between major races inhabiting large US cities. Specifically, we aim at answering the following questions. (1) How specific race subpopulations are redistributed across population zones of different racial characters? A notion of a population zone is shortly explained in section 2.2.2 and described in detail in Dmowska and Stepinski (2021). (2) Are those redistributions (called coresidence profiles) dependent on the geographical locations of cities across the US? We will show that there is indeed the geography of racial coresidence. (3) How coresidence patterns changed between 1990 and 2010?

To answer these questions we analyze 1990 and 2010 racial data for 61 largest cities across the US. Each city in each of the two years is divided into population zones. Next, for each combination of a city, racial group, and a year, we calculate a coresidence profile. The coresi- dence profile is the key concept of the presented analysis, it shows how a given racial subpopulation is distributed into different zones present in a given city at a given year. For example, how the Hispanic population is distributed into population zones in Houston, TX in 2010. This gives a deeper understanding of racial residential relations than existing metrics. To facilitate the US-wide analysis we cluster profiles of a given subpopulation from all cities in our sample into three archetype profiles. This reduction allows us to provide lucid answers to the three questions posed in the previous paragraph.

The paper is organized as follows. Data and methodology of establishing population zones are described in section 2 , followed by results in section 3 and discussion/conclusion in section 4 .

\section{Data and methods}

\subsection{Data}

We analyzed 61 most populous US metropolitan statistical areas (MSAs) in their 2010 borders, we refer to MSAs as "cities" while remembering that they are larger units. For these metropolitan areas, we used the 1990 and 2010 decennial census race/ethnicity data at the smallest aggregation level - that of the census block. We consider four subpopulations: White (W), Black (B), Asian (which include Hawaiian/Pacific Islanders) (A), and Hispanics $(\mathrm{H})$. All racial subpopulations are non-Hispanic and, in the rest of this paper, we refer to each subpopulation as a race for briefness while remembering that the Hispanics label refers to ethnicity.

\subsection{Method}

Our analysis consists of seven steps: (1) classifying census blocks into population types; (2) delineating zones as aggregates of given type blocks; (3) constructing coresidence profiles for zones; (4) clustering profiles from all cities for a given race and year into coresidence archetypes; (5) classifying cities on the basis of coresidence archetypes; (6) mapping the classified cities, and (7) comparing the mappings between 1990 and 2010 data. Step (1) and (2) are the preprocessing steps aiming at preparing data for further analysis; step (3) is performed to address the first aim of the paper, steps (4) to (6) address the second aim of this paper, and step (7) address the third aim of the paper. 

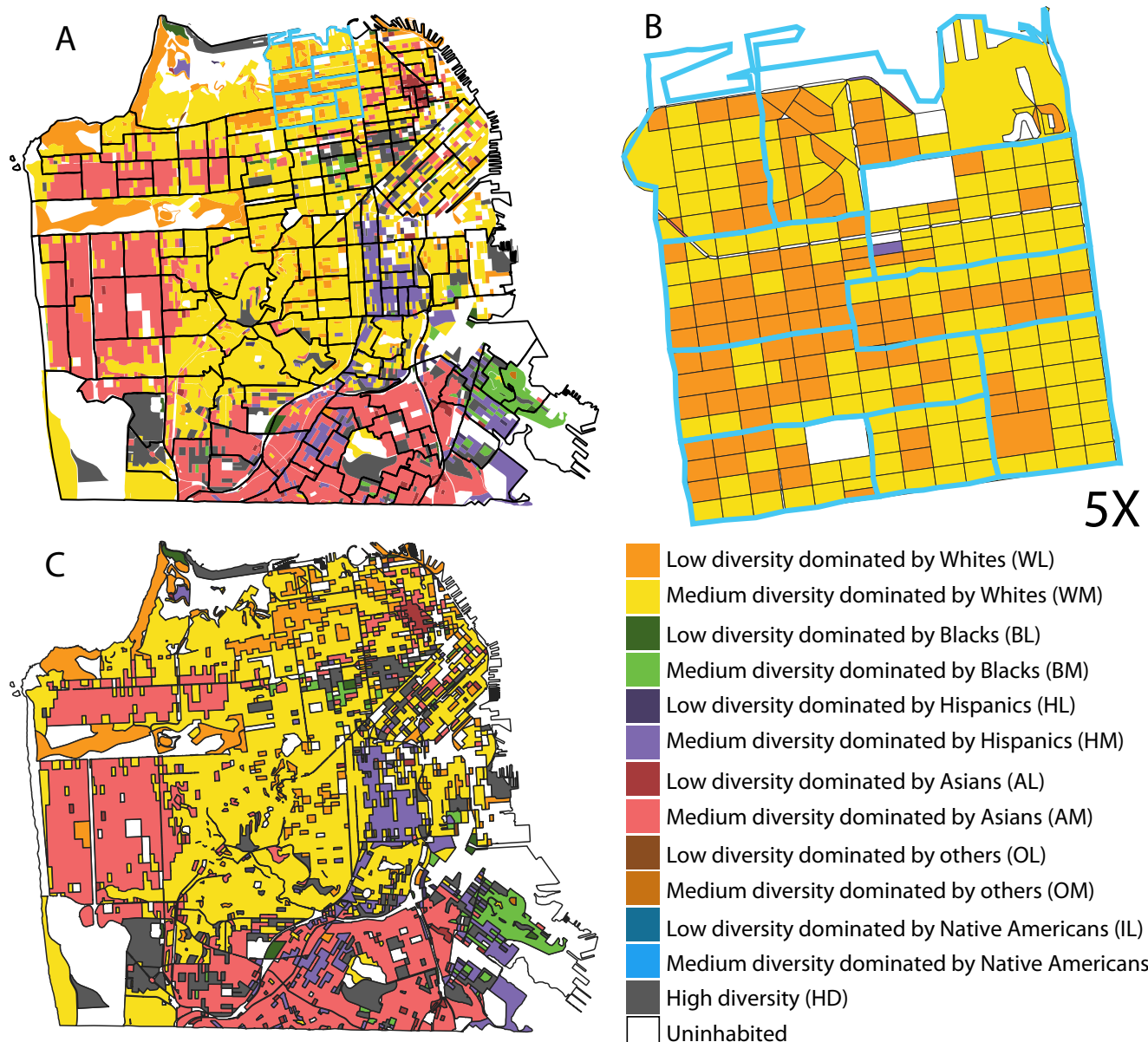

Low diversity dominated by Whites (WL) Medium diversity dominated by Whites (WM) Low diversity dominated by Blacks (BL) Medium diversity dominated by Blacks (BM) Low diversity dominated by Hispanics ( $\mathrm{HL}$ ) Medium diversity dominated by Hispanics (HM) Low diversity dominated by Asians (AL) Medium diversity dominated by Asians (AM) Low diversity dominated by others (OL) Medium diversity dominated by others (OM) Low diversity dominated by Native Americans (IL) Medium diversity dominated by Native Americans (IM) High diversity (HD) Uninhabited

Figure 1: Racial geography of San Francisco County is shown using different areal units. The colored background in all three panels is a map of population zones resulting from classifying 2010 census blocks into 13 types on the basis of racial diversity and the dominant race (see legend). (A) Boundaries of census tracts are superimposed on the racial map; many tracts are inhomogeneous with respect to population types. (B) A racial map of a small portion of San Francisco with boundaries of census tracts (blue) and census blocks (black) superimposed on the racial map; by construction, each block is assigned a single population type. (C) Boundaries of zones (see main text) are superimposed on the map of population zones; zones are homogeneous with respect to population types.

\subsubsection{Classifying census blocks into population types}

All inhabited blocks in each city are classified into 13 population types based on the level of diversity of their population and on an identity of the dominant race (Holloway et al., 2012; Dmowska et al., 2017). This classification scheme fits the US context and its associated civil rights legislation (Ellis et al., 2018). First, blocks are classified into three categories of diversity (low, medium, and high) on the basis of the value of the standardized entropy $(E)$ of their racial composition. Second, low and medium diversity blocks are further divided based on an identity of the dominant race, White (W), Black (B), Asian (A), Hispanics (H), Native Americans (I), and others (O). Thus, our final classi- fication of blocks consists of 13 types (White, low diversity (WL), Black, low diversity (BL), Hispanics, low diversity (HL), Asian, low diversity (AL), Native American, low diversity (IL), others, low diversity (OL), White, medium diversity (WM), Black, medium diversity (BM), Hispanics, medium diversity (HM), Asian, medium diversity (AM), Native American, medium diversity (IM), others, medium diversity (OM), high diversity (HD)).

\subsubsection{Delineating population zones}

It is important to stress that a division of a city should delineate racially (almost) homogeneous parts of the city as well as possible in order to not underes- 
timate a degree of segregation (Dmowska and Stepinski, 2021). This is best achieved by using the smallest aggregation units released by the Census Bureau - census blocks (see discussion in Dmowska and Stepinski (2021). Even if using blocks maximizes racially homogeneous parts of the city, many blocks remain racially inhomogeneous. The racial inhomogeneity of a block means that different races live together within. This is what we mean by the term "coresidence."

A zone of a given population type is an aggregate of all same-type blocks in the metropolitan areas. The aggregation is achieved using dissolve polygon algorithm provided by the GDAL/OGR library (GDAL/OGR, 2020). Zones, which inherit their diversity from constituent blocks, are more suitable than tracts for analyzing racial segregation (Chodrow, 2017; Stepinski and Dmowska, 2019; Dmowska and Stepinski, 2021) and for analyzing coresidence trends. Fig. 1 shows a division of San Francisco County into tracts (panel A), blocks (panel B), and population zones (C). The background is the block-based population types classification (see section 2.2.1). The purpose of this figure is to visually demonstrate that tracts are inhomogeneous but zones are homogeneous with respect to population types. Blocks are homogeneous too, but they are too small to constitute useful aggregation units. Note that in the present context, homogeneous means stationary - the racial composition is very similar in each block across the entire zone's extent. In each city, there are up to 13 different zones (one for each population type).

\subsubsection{Constructing coresidence profiles}

We use coresidence profiles to analyze the distribution of four major racial subpopulations (Whites, Blacks, Hispanics, and Asians) across nine major population type zones: WL, WM, BL, BM, HL, HM, AM, AL, HD. Population zones IL, IM, OL, OM consist of insignificant shares in the metropolitan areas and are omitted from our analysis. A coresidence profile is a property of a race, a city, and the year. For example, the coresidence profile for Asians in Chicago, in 1990 is as follows, (WL, 37\%), (WM, 44\%), (BL, 0.6\%), (BM, 2.5\%), (AL, 1.6\%), (AM, 5.7\%), (HL, 0.2\%), (HM, 5.6\%), and (HD, 2.8\%). Note that the majority of Asians in Chicago in 1990 lived in population zones dominated by Whites, and only the small minority of Asians lived in zones dominated by Asians. Such a profile can be presented graphically as a broken line connecting the nine points (zone, percentage). A collection of coresidence profiles for a given race (for example, Whites) in all 61 cities is referred to as a coresidence graph. Coresidence graphs for Whites, Blacks, Hispanics, and Asians are shown in Figs. 2A-5A for 1990, and in Figs. 2B-5B for 2010.

\subsubsection{Classifying cities based on coresidence profiles}

We cluster cities based on the similarity of their coresidence profiles for a given racial subpopulation in a given year. The purpose of clustering is to reduce the variety of coresidence profiles (each race has its own profile in each city) to a small number of archetype profiles to make the analysis more lucid. Based on experimentation, we had decided to cluster cities into three clusters (three archetypes). A single cluster groups cities with similar coresidence profiles for a given race and year. An average calculated from all profiles in a cluster is a single archetype profile that represents all cities in that cluster.

Note that clusters in 1990 are different from clusters in 2010. For reference purposes we ordered clusters from the largest to the smallest based on the number of cities they contain; the order of the cluster is its ID which is also used as an ID of corresponding archetype profile. For example, the largest cluster in 1990 pertaining to Whites has ID Whites $C 1_{1990}$. In coresidence graphs (Figs. 2A,B-5A,B) three different colors are used to draw 61 coresidence profiles to indicate their archetypes (clusters to which they belong). The colors for 1990 are different from colors in 2010 to underscore that archetypes are different each year.

Archetype profiles having one significant peak (for example, White $C 1_{1990}$ in Fig. 2A) groups cities where a given racial group live predominantly in monoracial setting (for example, zone WL). Clusters with archetype profiles having multiple significant peaks (for example, White $C 1_{2010}$ ) group cities where a given racial group lives in two different settings (for example, biracial zone WM and bi-racial zone $\mathrm{HM}$ ). The insets at the top of coresidence graphs (Figs. 2A,B-5A,B) give archetypes' profiles in the form of waffle charts instead of broken-lines.

Finally, we color-code cities based on a cluster ID to which they belong and plot them on the map of the US for visual analysis of the spatial correlation between similarity of coresidence profiles and geographical locations of cities. The maps are presented in Figs. 2C,D5C,D.

\section{Results}

Results are shown in Figs. 2-5. These four figures have the same structure consisting of two rows and two 

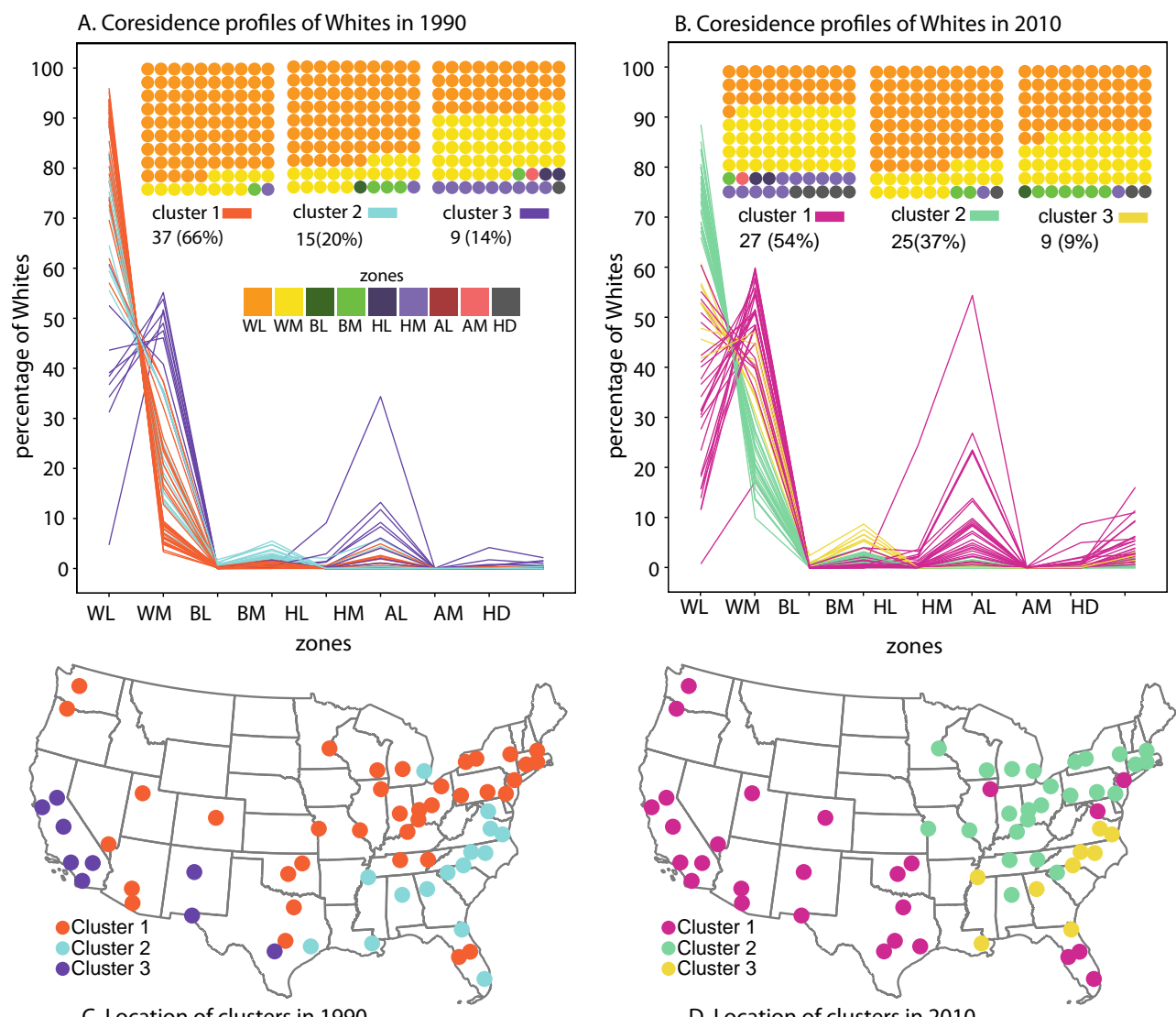

Figure 2: Coresidence of Whites in 61 cities. A) Coresidence profiles and their clustering in 1990. B) Coresidence profiles and their clustering in 2010. C) Geographical distribution of cities belonging to different coresidence clusters in 1990. D) Geographical distribution of cities belonging to different coresidence clusters in 2010. Insets at the top of panel A and B consist of waffle charts showing archetypes coresidence profiles calculated as averages from all profiles in corresponding clusters. The numbers below charts refer to the number of cities in a cluster. The numbers in the brackets refer to the percent of Whites aggregated over all cities living in the cluster.

columns but pertain to coresidence profiles of different races, White, Black, Asians, and Hispanics, respectively. The left column pertains to 1990 and the right column pertains to 2010. The top row shows profiles for all 61 cities to demonstrate that they indeed cluster into three groups. The three archetypes are shown as waffle charts and are marked by colors used to indicate cities in maps shown in the bottom row. Using these figures a reader can identify a specific archetype of coresidence for each city and observe the US-wide pattern coresidence profiles. Finally, the comparison of maps on the left and right informs about temporal changes in coresidence profiles. Numerical data for all coresidence profiles and the map with the names of all cities are provided in the Supplement.

Discussion of coresidence profiles shown in Figs. 2-5 calls for analysis from three different perspectives: (1) racial - each racial subpopulation has different coresi- dence profiles, (2) temporal - profiles changed between 1990 and 2010, and (3) geographical - even for a single racial subpopulation in a given year, coresidence profiles vary from one city to another; they are correlated with the racial composition of city's population, which, in turn, correlates with historical/geographical factors.

\subsection{Racial perspective}

Examining panels A and B in Figs. 2-5 we observe that coresidence profiles of all four racial groups in both years indeed cluster into no less than three archetypes. One could argue that, in some cases, more than three archetypes could be identified, but, in general, three archetypes are sufficient. This shows that although, in principle, each profile is unique in details, overall they can be grouped into a limited number of coresidence profile types (archetypes). 

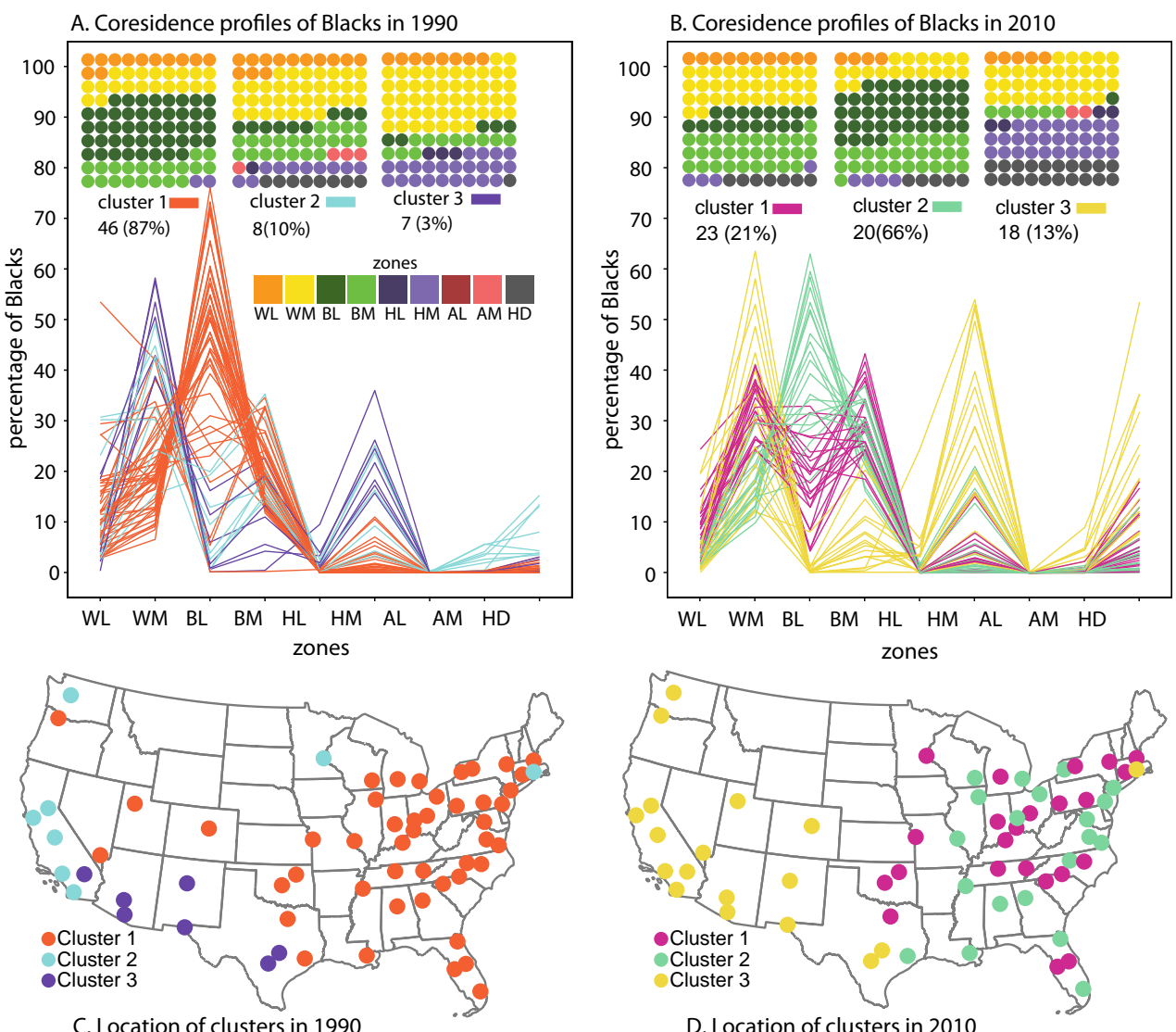

Figure 3: Coresidence of Blacks in 61 cities. A) Coresidence profiles and their clustering in 1990. B) Coresidence profiles and their clustering in 2010. C) Geographical distribution of cities belonging to different coresidence clusters in 1990. D) Geographical distribution of cities belonging to different coresidence clusters in 2010. Insets at the top of panel A and B consist of waffle charts showing archetypes coresidence profiles calculated as averages from all profiles in corresponding clusters. The numbers below charts refer to the number of cities in a cluster. The numbers in the brackets refer to the percent of Blacks aggregated over all cities living in the cluster.

All six archetypes (three for 1990 and three for 2010) for the White majority (waffle charts in Figs. 2A and B) show that Whites lived predominantly in either WL or WM zones. This may be attributed to the fact that at the beginning of the twentieth century the majority of US cities were practically all White and the segregation observed since that time is the result of an influx of people of different races. In addition, opinion surveys (Farley et al., 1978; Clark, 1992; Charles, 2000; Havekes et al., 2016) show that Whites prefer to live in neighborhoods with at least $50 \%$ of Whites and no more than $15 \%$ of Blacks or Hispanics. These preferences are in agreement with our empirical coresidence archetypes in both, 1990 and 2010.

Coresidence archetypes for Blacks (waffle charts in Figs. 3A and B) are more complex; there are no archetypes in which $50 \%$ or more Blacks live in BL zones. These archetypes are also in agreement with opinion surveys (Charles, 2000; Havekes et al., 2016) which show $37 \%$ of Blacks preferring to live in BM neighborhoods. Coresidence archetypes for Hispanics (waffle charts in Figs. 4A and B) show that in cities belonging to two archetypes the majority of Hispanics live in WM or WL zones, but there is also a group of cities where Hispanics live predominantly in HM and HD zones. Finally, coresidence archetypes for Asians (waffle charts in Figs. 5A and B) show that majority of them live in zones $\mathrm{WL}$ and $\mathrm{WM}$.

\subsection{Geographical perspective}

The geography of racial coresidence is shown in panels C and D in Figs. 2-5. The first observation is that indeed coresidence profiles of all racial groups correlate with geographical location. The second observation is that the particular dependence between a coresidence archetype and geographical region changes from one 

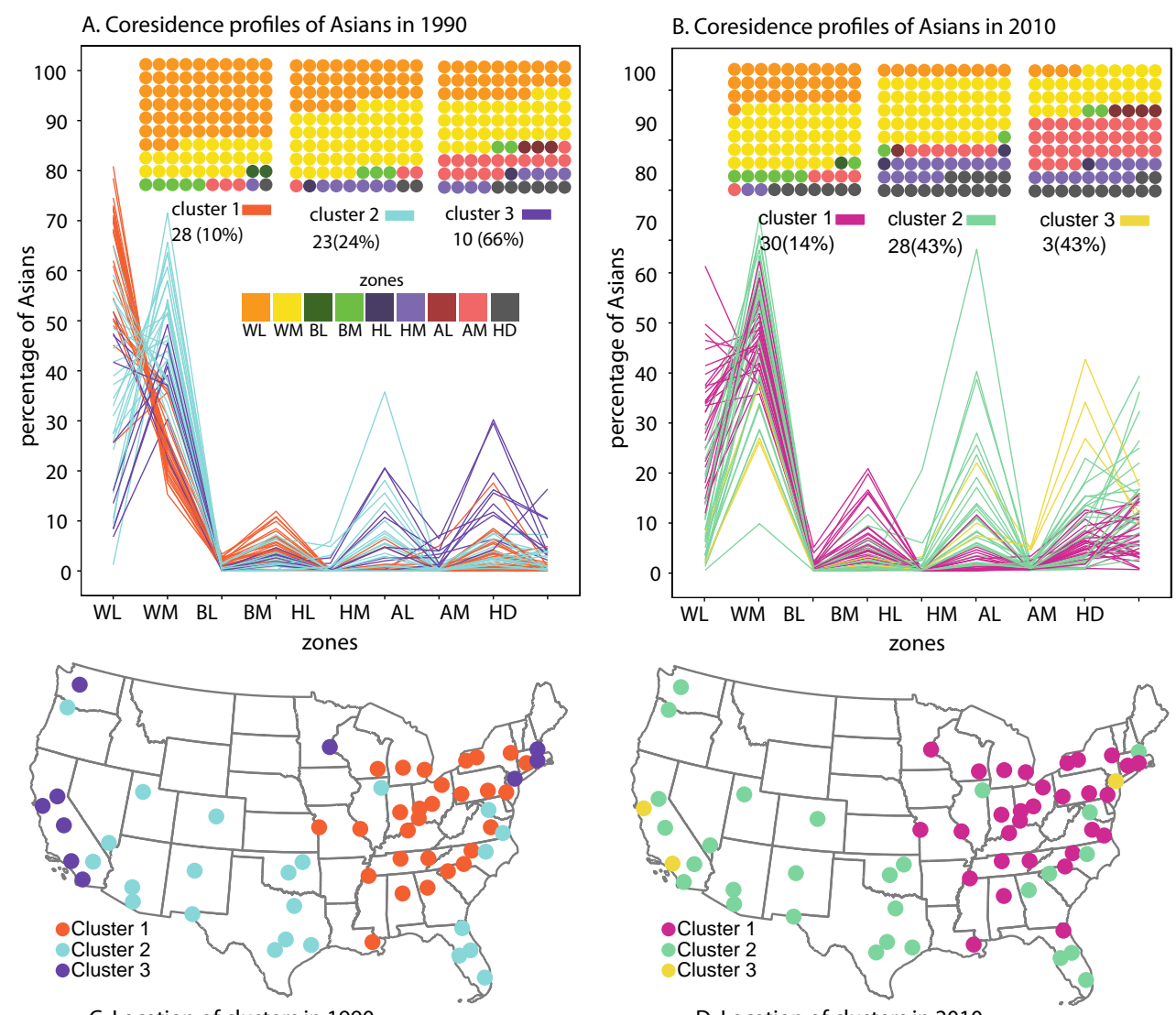

Figure 4: Coresidence of Asians in 61 cities. A) Coresidence profiles and their clustering in 1990. B) Coresidence profiles and their clustering in 2010. C) Geographical distribution of cities belonging to different coresidence clusters in 1990. D) Geographical distribution of cities belonging to different coresidence clusters in 2010. Insets at the top of panel A and B consist of waffle charts showing archetypes coresidence profiles calculated as averages from all profiles in corresponding clusters. The numbers below charts refer to the number of cities in a cluster. The numbers in the brackets refer to the percent of Asians aggregated over all cities living in the cluster.

subpopulation to another and from 1990 to 2010. For the purpose of discussion we will use a division of US into six geographic regions, Northeast (NE), Midwest (MW), Southeast (SE), Southwest (SW), Rocky Mountains (RM), and Pacific (P).

The map of coresidence profiles of Whites in 1990 is shown in Fig. 2C. In cities located in NE, MW (except Detroit), RM, and P (except California) the coresidence of Whites was characterized by archetype $C 1_{1990}$, Whites lived predominantly within their own race (zone WL). In most cities located in California, and SW the coresidence of Whites was characterized by archetype $C 3_{1990}$, most Whites lived in WM zones and significant percentage lived in HM zones. In most cities located in SE, the coresidence of Whites was characterized by archetype $C 2_{1990}$, most Whites lived in zone WL, but a significant percent of Whites lived in the WM zone, and some lived in the BM zone. Most of these geographical regularities may be explained in terms of availability of minority population, with Hispanics available in California and SW and Blacks available in SE. Comparison of Fig. 2C and 2D indicates that the geographical distribution of coresidence profiles of Whites underwent some changes between 1990 and 2010, however, the major geographical division based on the availability of minority groups remained the same. The changes may be attributed to an increased population of Hispanics.

The map of coresidence profiles of Blacks in 1990 is shown in Fig. 3C. In most cities located in NE, MW (except Detroit), SE, and RM, the coresidence of Blacks were characterized by the archetype $C 1_{1990}$. In these cities, almost half of the total Black population lived in the monoracial BL zone. The remaining share of the Black population lived predominantly in BM and WM zones (both mixtures of White and Black residences), and a small share lived in the WL zone. In cities lo- 

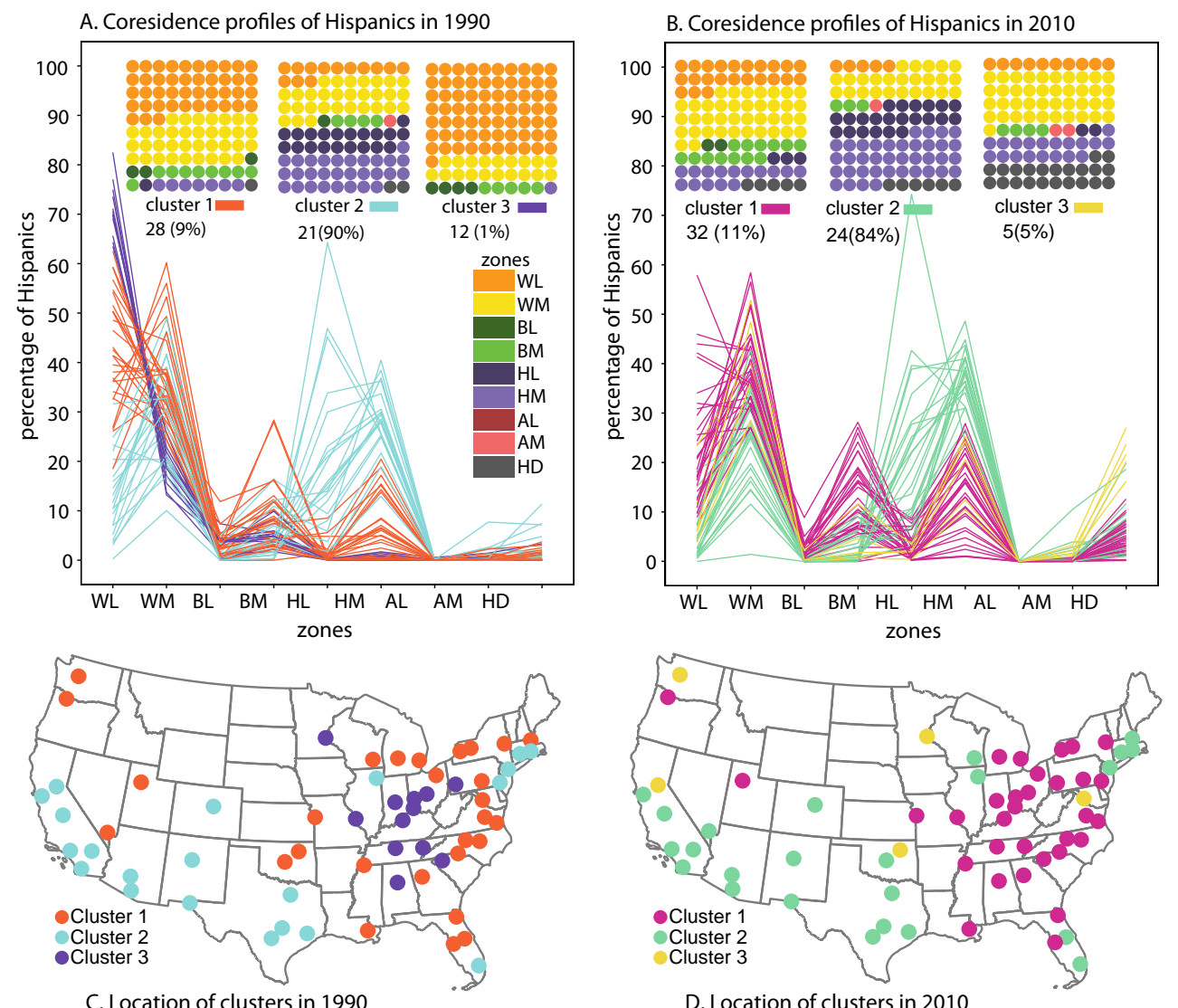

Figure 5: Coresidence of Hispanics in 61 cities. A) Coresidence profiles and their clustering in 1990. B) Coresidence profiles and their clustering in 2010. C) Geographical distribution of cities belonging to different coresidence clusters in 1990. D) Geographical distribution of cities belonging to different coresidence clusters in 2010. Insets at the top of panel A and B consist of waffle charts showing archetypes coresidence profiles calculated as averages from all profiles in corresponding clusters. The numbers below charts refer to the number of cities in a cluster. The numbers in the brackets refer to the percent of Hispanics aggregated over all cities living in the cluster.

cated in California plus three cities outside of California the coresidence of Blacks was characterized by the archetype $C 2_{1990}$. About half of the Black population in these cities lived in either WM or WL zones and another half in multiple different zones; a small share of the Black population lived in the BL zone. The geography of coresidence of Blacks changed from 1990 to 2010. In 2010, the coresidence profile of Blacks in cities located in the western part of the US (P, RM, and SW) was described by the archetype $C 3_{2010}$; Blacks lived distributed between other races. In the easter part of the US, coresidence of Blacks was a mixture between $C 2_{2010}$ (more segregated from other groups) and $C 1_{2010}$ (less segregated from other groups).

The map of coresidence profiles of Asians in 1990 is shown in Fig. 4C. Most cities located in NE, MW, and $\mathrm{SE}$ (except Florida) were characterized by the coresidence archetype $C 1_{1990}$; Asians lived predominantly in the WL zone, while the second-largest share of Asians lived in the WM zone. Most cities located in SW, RM, and Florida were characterized by the coresidence archetype $C 2_{1990}$; Asians lived predominantly in the WM zone, while the second-largest share of Asians lived in the WL zone. Cities located in P plus three large cities in NE plus Minneapolis were characterized by the coresidence archetype $C 3_{1990}$; most Asian lived WM and WL zones, but a significant share of Asian lived in the AM zone. During the 1990-2010 period, the geography of the coresidence of Asians had changed little but the archetype underwent a significant change.

The map of coresidence profiles of Hispanics in 1990 is shown in Fig. 5C. Most cities located in SW, RM, California, and coastal cities in Florida and NE were characterized by the coresidence archetype $C 2_{1990}$; about half of the Hispanic population lived in zones HM and HD, and the other half in zones WM and WL. There was 


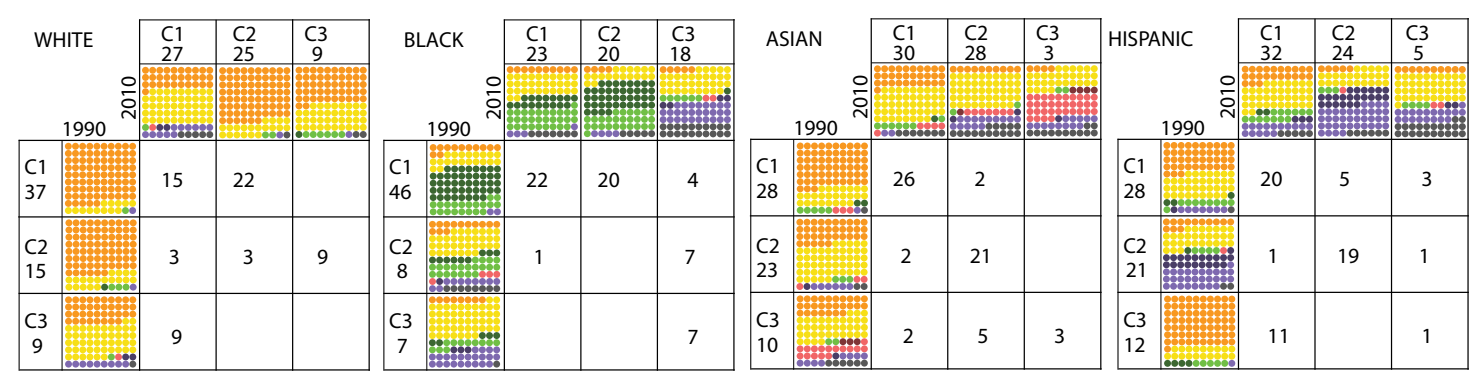

Figure 6: Transition matrix illustrating a number of MSAs transitioning from 1990 coresidence archetypes to 2010 archetypes.

a cluster of cities located mostly in MW characterized by the coresidence archetype $C 3_{1990}$; Hispanics lived mostly among Whites. In the remaining cities, scattered across the US Hispanics lived mostly among Whites, but a notable percentage of Hispanics lived in the zone HM. During the 1990-2010 period, the geography of coresidence of Hispanics had changed. The first group of cities preserved its archetype but with an increased share of Hispanics in zones HM and HD. The second and third groups of cities merged into the archetype $C 1_{2010}$, and the new archetype, $C 3_{2010}$ has formed.

\subsection{Temporal perspective}

Fig. 6 shows transition matrices illustrating changes in coresidence profiles during the 1990-2010 period. Analyzing racial segregation change using transition matrix was previously used by Fasenfest et al. (2004), Logan and Zhang (2010), Farrell and Lee (2011), and Wright et al. (2018) among others, although they used it to show changes in tract classification and we use it to show changes in coresidence profiles. waffle charts in the second column and the second row of transition matrices show cluster-averaged coresidence profiles (archetypes) in 1990 and 2010, respectively. These are the same waffle charts having been shown as inserts in Fig. 2A,B-5A,B. Numbers in the matrix indicate how many cities having a particular type of coresidence profile in 1990 transitioned to different profiles in 2010. For example, of the 46 cities having the Black $C 1_{1990}$ profile, 22 cities transitioned to the Black $C 1_{2010}$ profile, 20 cities transitioned to the Black $C 2_{2010}$ profile, and 4 cities transitioned to the Black $C 3_{2010}$ profile. The transition matrix illustrates how many cities have changed their coresidence profiles to become more diverse, less diverse or to maintain their diversity during the 19902010 period.

The transition matrix for Whites shows three transitions that did not result in a significant change in coresidence profiles of Whites. For example, the $C 1_{1990} \rightarrow$
$C 2_{2010}$ transition (22 cities colored orange in Fig. 2C and colored green in Fig. 2D) preserves the mostly White monoracial profile. Other no-change or small change transitions are $C 2_{1990} \rightarrow C 2_{2010}$ and $C 3_{1990} \rightarrow$ $C 1_{2010}$. The remaining three transitions lead to a significant change in the coresidence profiles of Whites. For example, the $C 1_{1990} \rightarrow C 1_{2010}$ transition (15 cities colored orange in Fig. 2C and colored violet in Fig. 2D) resulted in the change from mostly monoracial settings to predominantly bi-racial settings. Other change transitions are $C 2_{1990} \rightarrow C 1_{2010}$ and $C 2_{1990} \rightarrow C 3_{2010}$.

The transition matrix for Blacks shows three transitions that did not result in a significant change in coresidence profiles of Blacks. For example, the $C 1_{1990} \rightarrow$ $C 2_{2010}$ transition (20 cities colored orange in Fig. 3C and colored green in Fig. 3D) preserves the profile in which almost half of Blacks live in a monoracial zone BL, while approximately a quoter of Blacks lives in a biracial zone BM. Other no-change or small change transitions are $C 2_{1990} \rightarrow C 1_{2010}$ and The $C 3_{1990} \rightarrow C 3_{2010}$. The remaining three transitions lead to a significant change in coresidence profiles of Blacks. For example, the $C 1_{1990} \rightarrow C 1_{2010}$ transition (22 cities colored orange in Fig. 3C and colored violet in Fig. 3D) resulted in a significant decrease of Blacks living in the BL zone and an increase in Blacks living in the BM and WM (bi-racial) zones. Other change transitions are $C 1_{1990} \rightarrow C 3_{2010}$ and $C 2_{1990} \rightarrow C 3_{2010}$.

The transition matrix for Asians shows seven different transitions of which one, $C 2_{1990} \rightarrow C 1_{2010}$, resulted in a relatively small change and the rest resulted in a significant change. The transition matrix for the Hispanic population shows eight different transitions of which three resulted in a relatively small change and five resulted in a larger change. The small change transitions are $C 1_{1990} \rightarrow C 1_{2010}, C 2_{1990} \rightarrow C 2_{2010}$, and $C 2_{1990} \rightarrow C 3_{2010}$. The remaining resulted in a significant change. It is beyond the scope of this paper to describe all transitions in detail, however, the examination 
Table 1: Shares of race-specific population living in the zones of a given diversity/dominant type

\begin{tabular}{|c|c|c|c|c|c|c|c|c|c|c|}
\hline & Zones & WL & WM & BL & BM & $\mathbf{A L}$ & $\mathbf{A M}$ & HL & HM & HD \\
\hline \multirow{3}{*}{ White } & 1990 & 76.0 & 19.6 & 0.3 & 1.4 & 0.0 & 0.3 & 0.3 & 1.8 & 0.3 \\
\hline & 2010 & 52.6 & 36.7 & 0.2 & 2.0 & 0.0 & 0.9 & 0.5 & 4.2 & 2.9 \\
\hline & change & -23.4 & 17.1 & -0.1 & 0.6 & 0.0 & 0.6 & 0.2 & 2.4 & 2.6 \\
\hline \multirow{3}{*}{ Black } & 1990 & 6.5 & 16.6 & 49.2 & 20.8 & 0.0 & 0.4 & 0.4 & 4.9 & 1.2 \\
\hline & 2010 & 3.7 & 19.8 & 30.7 & 26.9 & 0.0 & 0.8 & 0.9 & 9.7 & 7.5 \\
\hline & change & -2.8 & 3.2 & -18.5 & 6.1 & 0.0 & 0.4 & 0.5 & 4.8 & 6.3 \\
\hline \multirow{3}{*}{ Asian } & 1990 & 25.2 & 41.5 & 0.6 & 3.3 & 2.0 & 13.2 & 0.9 & 9.3 & 4.0 \\
\hline & 2010 & 9.1 & 39.9 & 0.3 & 2.8 & 2.4 & 19.8 & 0.9 & 10.5 & 14.3 \\
\hline & change & -16.1 & -1.6 & -0.3 & -0.5 & 0.4 & 6.6 & 0.0 & 1.2 & 10.3 \\
\hline \multirow{3}{*}{ Hispanic } & 1990 & 12.0 & 26.1 & 1.2 & 5.4 & 0.0 & 1.3 & 22.9 & 29.5 & 1.6 \\
\hline & 2010 & 5.1 & 22.9 & 0.7 & 4.8 & 0.0 & 1.7 & 23.2 & 35.1 & 6.5 \\
\hline & change & -6.9 & -3.2 & -0.5 & -0.6 & 0.0 & 0.4 & 0.3 & 5.6 & 4.9 \\
\hline
\end{tabular}

of Fig. 6 provides all information to interpret all transitions. To put these transitions into geographical context, the cities corresponding to entries in transition matrices can be identified on maps (Figs. 2C,D-5C,D).

Table 1 gives numerical values of coresidence profiles constructed from pooling data from all 61 cities in our sample. Profiles in Table 1 are in the format somewhat comparable with the format of previously published results (Fasenfest et al., 2004; Farrell and Lee, 2011) except that Table 1 gives percentages of inhabitants and pertains to the 1990-2010 period whereas previously published pertained to the $1990-2000$ period and, in the majority of cases, listed the percentage of neighborhoods. Also, the classification of neighborhoods is different in each study.

\section{Conclusion and discussion}

The topic of residential race relations in US cities has been addressed in numerous studies, but predominantly from a single perspective, that of racial segregation. This perspective fitted the situation when cities were biracial and sharply segregated. Today, US cities are racially diverse (multiracial) and less sharply divided along racial lines. Metrics of segregation were updated to account for that change, but in the process, they become rather abstract. In this paper, we proposed a different analytical perspective; instead of quantifying segregation, we quantify coresidence between different subpopulations. This who-lives-with-whom perspective provides less abstract and a more concrete description of residential race relations. What distinguishes our work from past reports is a direct coresidence approach based on counting people directly instead of counting neighborhoods, and counting people in each city separately instead of using data pooled from all cities in the sample as was done in Fasenfest et al. (2004) and Farrell and Lee (2011).

Another distinction is the presentation of the USwide geography of coresidence. This demonstrates that coresidence profiles are correlated with the geography of race. It was previously thought that coresidence profiles are mostly due to racial preferences, and in fact, the most popular model of racial segregation (Schelling, 1971) is based on racial preferences. Our findings do not preclude the existence of preferences but indicate that they are strongly modulated by the availability of racial subpopulations. For example, in California, where the Hispanic subpopulation was already large in 1990, the coresidence profiles of Whites and Blacks are very different from what they were in NE and MW, where the subpopulation of Hispanics was low in 1990. In 2010, when the subpopulation of Hispanics become larger in NE and MD cities, the coresidence profiles of Whites and Blacks started to show more mixing with Hispanics. When the 2020 US census block-based data becomes available, we would repeat the analysis presented in this paper for the 2010-2020 period. As we expect a further expansion of the Hispanic subpopulation, our hypothesis of the dependence of coresidence profiles on the availability of racial groups will be tested.

Based on our results we can now answer three questions posed in the introductory section.

How race subpopulations are redistributed across zones of different racial characters? Panels A and B in Figs. 2-5 provide answers to this question. There is no need to describe a coresidence profile of a given group separately for each city. Instead, all profiles fall into one of three archetypes, these archetypes are characterized by waffle charts shown as insets in Figs. 2A,B-5A,B. 
Are coresidence profiles correlated with geography? Yes, they are. Panels C and D in Figs. 2-5 show locations of cities in our sample colored according to an archetype of coresidence profile. Spatial dependence is observed. We submit that this dependence follows mostly from the availability of specific minority races to share places of residence with the majority White population.

How coresidence patterns changed between 1990 and 2010? The answer to this question is obtained by visually comparing panels $\mathrm{C}$ with panels D on Figs. 25. The quantitative answer is provided in Fig. 6, where numbers of transitions from archetypes in 1990 to archetypes in 2010 are listed. Table 1 provides a quantitative assessment of change for the pool of data from all cities in the sample.

Some questions may arise with respect to our use of population zones. How dependent are spatial extents of zones on the values of thresholds used in the classification of blocks? Are extents of zones sensitive to the boundaries of the blocks? Why the zones are particularly well-suited for our analysis? These questions have been answered in the previous works (Holloway et al., 2012; Dmowska and Stepinski, 2021), here we shortly summarize the arguments.

In principle, a large change of thresholds would change the zones, however, such change would be unjustified. The values of parameters set by Holloway et al. (2012) fit the US context and correspond to civil rights legislation (Ellis et al., 2018). The validity of these threshold values can be checked by visually comparing the zones in San Francisco County (Fig. 1C) with a one-dot-per-person map of the county (http://racialdotmap.demographics.coopercenter.org/).

From such comparison, it is clear that zones reflect well the spatial changes in population mix. From the map of zones or the dot map it also can be observed that the characteristic size of population mixes (zones) is larger than the characteristic size of a single block. Thus, delineating zones from a hypothetical, alternative delineation of blocks will not significantly change the zones. Finally, zones are particularly well-suited for our purpose because they are large units of homogeneous population type. Dmowska and Stepinski (2021) shown that homogeneity of zones with respect to population types is larger than homogeneity of much smaller census tracts.

Comparison to previous work can only be done qualitatively because of different methodologies and different time periods. Results from previous works could be summarized as follows. (1) The number of White-dominated neighborhoods is declining with time. (2) The number of mixed (bi-racial or multiracial) neighborhoods is increasing with time. (3) Mixed, White-other (White-Asian, White-Hispanic) neighborhoods are more stable than White-Black neighborhoods. These results pertain to studies covering mostly the 1990-2000 period, although Logan and Zhang (2010) covered the 1980-2000 period and Wright et al. (2018) covered the 1990-2010 period. The present analysis (pertaining to the 1990-2010 period) is in qualitative agreement with the first two conclusions from previous works. This is best seen from Table 1 which shows steep decreases in the percentage of the population living in the WL zone across all racial groups and an increase in the percentage of the White and Black population living in the WM zone. Other bi-racial zones saw moderate (both positive as well as negative) changes in the percentage of the population living there. The HD zone saw an increase in the percentage of the population across all racial groups. However, the present study is not designed to analyze the stability of coresidence because it is based only on one temporal period.

\section{References}

Bader, M. D., Warkentien, S., 2016. The fragmented evolution of racial integration since the civil rights movements. Sociological Science 3, 133-166.

Bellman, B., Spielman, S. E., S., R., Franklin, 2018. Local Population Change and Variations in Racial Integration in the United States, 2000-2010. International regional science review 41(2), 233-255.

Charles, C. Z., 2000. Neighborhood racial-composition preferences: Evidence from a multiethnic metropolis. Social problems 47(3), 379-407.

Chodrow, P. S., 2017. Structure and information in spatial segregation. Proceedings of the National Academy of Sciences 114(44), 1159111596.

Clark, W. A., 1992. Residential preferences and residential choices in a multiethnic context. Demography 29(3), 451-466.

Clark, W. A., Anderson, E., Östh, J., Malmberg, B., 2015. A Multiscalar Analysis of Neighborhood Composition in Los Angeles, 20002010: A Location-Based Approach to Segregation and Diversity. Annals of the Association of American Geographers 105(6), pp.1260-1284. 105(6), 1260-1284.

Dmowska, A., Stepinski, T. F., 2021. Improving assessment of urban racial segregation by partitioning a region into racial enclaves. Environment and Planning B: Urban Analytics and City Science, Doi 23998083211001386

URL https : //doi.org/10.1177/23998083211001386

Dmowska, A., Stepinski, T. F., Netzel, P., 2017. Comprehensive framework for visualizing and analyzing spatio-temporal dynamics of racial diversity in the entire United States. PLoS ONE 12(3), e0174993.

Ellis, M., Wright, R., Holloway, S., Fiorio, L., 2018. Remaking white residential segregation: metropolitan diversity and neighborhood change in the United States. Urban Geography 39(4), 519-545.

Farley, R., Schuman, H., Bianchi, S., Colasanto, D., Hatchett, S., 1978. Chocolate city, vanilla suburbs: Will the trend toward racially separate communities continue? Social Science Research 7(4), .319-344. 
Farrell, C. R., 2008. Bifurcation, fragmentation or integration? The racial and geographical structure of US metropolitan segregation, 1990-2000. Urban Studies 45, 467-499.

Farrell, C. R., Lee, B. A., 2011. Racial diversity and change in metropolitan neighborhoods. Social Science Research 40 (4), 1108-1123.

Fasenfest, D., Booza, J., Metzger, K., 2004. Living Together : A New Look at Racial and Ethnic Integration in Metropolitan Neighborhoods, 1990-2000. Tech. rep.

Fischer, C. S., Stockmayer, G., Stiles, J., Hout, M., 2004. Distinguishing the geographic levels and social dimensions of US metropolitan segregation, 1960-2000. Demography 41(1), 37-59.

Fischer, M. J., 2003. The relative importance of income and race in determining residential outcomes in US urban areas, 1970-2000. Urban Affairs Review 38(5), 669-696.

Fowler, C. S., Lee, B. A., Matthews, S. A., 2016. The contributions of places to metropolitan ethnoracial diversity and segregation: Decomposing change across space and time. Demography 53(6), 1955-1977.

Frey, W. H., 2004. The new great migration: Black Americans' return to the South, 1965-2000. Center on Urban and Metropolitan Policy, the Brookings Institution.

GDAL/OGR, 2020. GDAL/OGR contributors. gdal/ogr geospatial data abstraction software library. open source geospatial foundation. https://gdal.org.

Havekes, E., Bader, M., Krysan, M., 2016. Realizing racial and ethnic neighborhood preferences? Exploring the mismatches between what people want, where they search, and where they live. Population Research and Policy Review 35(1), 101-126.

Hoeffel, E. M., Rastogi, S., Kim, M. O., Hasan, S., 2012. The Asian population: 2010. US Department of commerce, economics and statistics administration, US Census Bureau.

Holloway, S. R., Wright, R., Ellis, M., 2012. The racially fragmented city? Neighborhood racial segregation and diversity jointly considered. The Professional Geographer 64, 63-82.

Hong, S. Y., O'Sullivan, D., Sadahiro, Y., 2014. Implementing spatial segregation measures in R. PloS one 9(11), e113767.

Humes, K. R., Jones, N. A., Ramirez, R. R., 2011. Overview of race and Hispanic origin: 2010. US Department of commerce, economics and statistics administration, US Census Bureau.

Iceland, J., 2004. Beyond Black and White metropolitan residential segregation in multi-ethnic America. Social Science Research 33 , 248-271.

Iceland, J., Weinberg, D., Steinmetz, E., 2002. Racial and Ethnic Residential Segregation in the United States: 1980-2000. Tech. rep.

Logan, J. R., Stults, B. J., Farley, R., Stults, J., 2004. Segregation of minorities in the metropolis: Two decades of change. Demography 41 (1), 1-22.

Logan, J. R., Zhang, C., 2010. Global neighborhoods: New pathways to diversity and separation. American Journal of Sociology 115 (4), 1069-1109.

Massey, D. S., Denton, N. A., 1987. Trends in the residential segregation of blacks, hispanics, and asians: 1970-1980. American sociological review, 802-825.

Parisi, D., Lichter, D. T., Taquino, M. C., 2011. Multi-scale residential segregation: Black exceptionalism and America's changing color line. Social Forces 89(3), 829-852.

Schelling, T. C., 1971. Dynamic models of segregation. Journal of Mathematical Sociology 1, 143-186.

Stepinski, T. F., Dmowska, A., 2019. Imperfect melting pot-Analysis of changes in diversity and segregation of US urban census tracts in the period of 1990-2010. Computers, Environment and Urban Systems 76, 101-109.

Wright, R., Ellis, M., Holloway, S. R., Catney, G., 2018 The instability of highly racially diverse residential neighbor- hoods in the united states. Sociology of Race and Ethnicity, 2332649218819168.

Wright, R., Ellis, M., Holloway, S. R., Wong, S., 2014. Patterns of racial diversity and segregation in the United States: 1990-2010. The Professional Geographer 66(2), 173-182.

Yao, J., Wong, D. W., Bailey, N., Minton, J., 2019. Spatial segregation measures: A methodological review. Tijdschrift voor economische en sociale geografie 110(3), 235-250. 